\title{
Large volume behaviour of Yang-Mills propagators
}

\section{Christian S. Fischer*}

Institut für Physik, Technische Universität Darmstadt, Schlossgartenstr. 9, 64289 Darmstadt, Germany

E-mail: christian.fischer@physik.tu-darmstadt.de

\section{Reinhard Alkofer}

Institut für Physik, Karl-Franzens-Universität, Universitätsplatz, 5, A-8010 Graz, Austria

\section{Axel Maas}

Institute of Physics, Slovak Academy of Sciences, Dúbravská cesta 9,

SK-845 11 Bratislava, Slovakia

\section{Jan M. Pawlowski}

Institut für Theoretische Physik, University of Heidelberg, Philosophenweg 16, 62910 Heidelberg, Germany

\section{Lorenz von Smekal}

Centre for the Subatomic Structure of Matter, School of Chemistry and Physics,

The University of Adelaide, SA 5005, Australia

\begin{abstract}
We summarise results on finite-volume effects in the propagators of Landau gauge Yang-Mills theory using Dyson-Schwinger equations on a 4-dimensional torus. We demonstrate explicitly how the solutions for the gluon and the ghost propagator tend towards their respective infinite volume forms in the corresponding limit. We discuss the relation of our solutions with results from lattice Monte-Carlo simulations.
\end{abstract}

The XXV International Symposium on Lattice Field Theory

July 30-4 August 2007

Regensburg, Germany

\footnotetext{
${ }^{*}$ Speaker.
} 


\section{Introduction}

In Landau gauge continuum Yang-Mills theory there are two confinement scenarios connected to the infrared behaviour of the gluon and ghost propagators: the Kugo-Ojima scenario [1] and the Gribov-Zwanziger picture [2]. Both predict an infrared enhanced ghost propagator and an infrared suppressed gluon propagator (for reviews see [3]). In the Kugo-Ojima scenario BRSTsymmetry is used to define a physical subspace of BRST-singlets within the complete state space of covariant gauge QCD. Provided global gauge symmetry is unbroken one can show that the space of BRST-singlets indeed contains colourless objects only. This condition is connected to the infrared behaviour of the ghost dressing function in Landau gauge QCD: The global colour charge is well-defined if and only if the ghost dressing function is singular in the infrared. This so called Kugo-Ojima condition is necessary, but not sufficient, for confinement in the Kugo-Ojima scenario. The Gribov-Zwanziger scenario, on the other hand, postulates that gauge field configurations on the Gribov-horizon are responsible for the confining nature of the quark-antiquark potential. In terms of Green's functions these gauge field configurations have been identified to induce infrared enhancement in the ghost and infrared suppression of the gluon propagator [2, 4, 5].

Studies of (untruncated) Dyson-Schwinger equations (DSE) and functional renormalisation group equations (FRGE) in the infinite volume/continuum limit strongly support these scenarios. In Landau gauge the ghost and gluon propagators are given by

$$
D^{G}\left(p^{2}\right)=-\frac{G\left(p^{2}\right)}{p^{2}}, \quad D_{\mu v}\left(p^{2}\right)=\left(\delta_{\mu v}-\frac{p_{\mu} p_{v}}{p^{2}}\right) \frac{Z\left(p^{2}\right)}{p^{2}}
$$

where $G\left(p^{2}\right)$ denotes the ghost dressing function and $Z\left(p^{2}\right)$ the dressing function of the gluon. One finds [6] that the small momentum behaviour of these functions is given by power laws, i.e.

$$
G\left(p^{2}\right) \sim\left(p^{2}\right)^{-\kappa}, \quad Z\left(p^{2}\right) \sim\left(p^{2}\right)^{2 \kappa} .
$$

Here the exponents of ghost and glue are uniquely related by the anomalous dimension $\kappa$. Similar expressions have been found for all other one-particle irreducible (1PI) Greens's functions in Landau gauge [7, 8]; see section 2. In the notation (1.2) the Kugo-Ojima and Gribov-Zwanziger scenarios translate to the condition $\kappa \geq 1 / 2$. Indeed, in the DSE and FRGE approaches one obtains $\kappa=(93-\sqrt{1201}) / 98 \approx 0.595[4,9,10]$, which satisfies both criteria.

On the lattice the verification of the relations (1.2) in gauge fixed calculations turned out to be extremely cumbersome, for latest results see $[11,12,13,14,15,16,17,18,19,20]$. To extract information on the power laws (1.2) from studies on a finite volume it is most important to address the volume dependence of the long-range behaviour of these correlations. It is only when this dependence is under control that firm conclusions can be drawn from extrapolations to the infinite volume and continuum limits.

In order to understand possible patterns of such volume effects it is an obvious and necessary step forward to adapt the continuum methods to finite volumes [21, 22]. We summarise these efforts in section 3 and compare the results to available lattice data. 


\section{Infrared exponents of 1PI Green's functions}

The infrared behaviour of the one-particle irreducible (1PI) Green's functions of Yang-Mills theory have been investigated in a number of works. The basic relation (1.2) between the dressing functions of the gluon and ghost propagator has been extracted first in [6]. These findings have been generalised to Green's functions with an arbitrary number of legs in [7]. The analysis rests upon a separation of scales, which takes place in the deep infrared momentum region. Provided there is only one external momentum $p^{2}$ much smaller than $\Lambda_{\mathrm{QCD}}$, a self-consistent infrared asymptotic solution of the whole tower of DSEs or FRGEs for these functions is given by

$$
\Gamma^{n, m}\left(p^{2}\right) \sim\left(p^{2}\right)^{(n-m) \kappa} .
$$

Here $\Gamma^{n, m}\left(p^{2}\right)$ denotes the dressing function of the infrared leading tensor structure of the 1PIGreen's function with $2 n$ external ghost legs and $m$ external gluon legs. It is important to note that the solution (2.1) is unique [8] which follows from a comparison of untruncated towers of DSEs and FRGEs. The exponent $\kappa$ is known to be positive [23, 9]. We emphasise that these findings do not rely on any truncation scheme.

This specific value of $\kappa=(93-\sqrt{1201}) / 98 \approx 0.595$, however, depends on the assumption that a bare ghost-gluon vertex is a good approximation to the full vertex in the infrared. This assumption has been tested in the continuum [24] and on the lattice [18, 25], and found to be adequate. Possible corrections by regular dressings in the infrared have been investigated within the DSE framework in [9], where a possible range $0.5 \leq \kappa<0.7$ has been given. Note that the value $\kappa=1 / 2$ marks the watershed between an infrared vanishing $(\kappa>1 / 2)$ and an infrared divergent $(\kappa<1 / 2)$ gluon propagator. The first option necessarily entails that the gluon propagator violates positivity as can be seen from

$$
0=D(p=0)=\int d^{4} x \Delta(x),
$$

with $D(p)=Z\left(p^{2}\right) / p^{2}$. This relation implies that the propagator function in coordinate space, the Schwinger function $\Delta(x)$, must contain positive as well as negative norm contributions, with equal integrated strengths. Therefore infrared vanishing gluons cannot be part of the positive definite, physical state space of Yang-Mills theory. While the infrared vanishing of the gluon propagator is not at all necessary for the positivity violations of transverse gluons, one needs to analyse the convexity in Euclidean time of its one-dimensional Fourier transform to establish this, if the gluon is infrared finite or even divergent.

Further interesting consequences of the solution (2.1) are the existence of infrared fixed points in the running couplings of Yang-Mills theory $[6,7]$.

\section{Dyson-Schwinger equations on a torus}

There are several caveats in comparing results from the continuum Dyson-Schwinger approach to those of lattice calculations. First, the quantitative aspects of the continuum solutions (i.e. the value of $\kappa$ and numerical results at intermediate momenta) depend on the details of the chosen truncation scheme, whereas the lattice calculations are ab initio. On the other hand, simulations are performed on finite lattices and one has to deal with the effects due to the finiteness of volume and lattice spacing. 
To quantify the 'plain' volume effects (i.e. those not connected to the gauge fixing procedure) we formulated the DSEs on a torus [21,22], employing the same truncation scheme as in the infinite volume/continuum framework [26]. One would then expect to see differences to the continuum solution for small volumes, which disappear continuously when the volume is chosen larger and larger. This expectation is supported by the FRGE infrared studies with an explicit infrared cut-off [10]. In earlier works on DSEs on the torus [21] this issue could not be resolved but an improved finite-volume renormalisation procedure indeed led to a smooth transition [22].
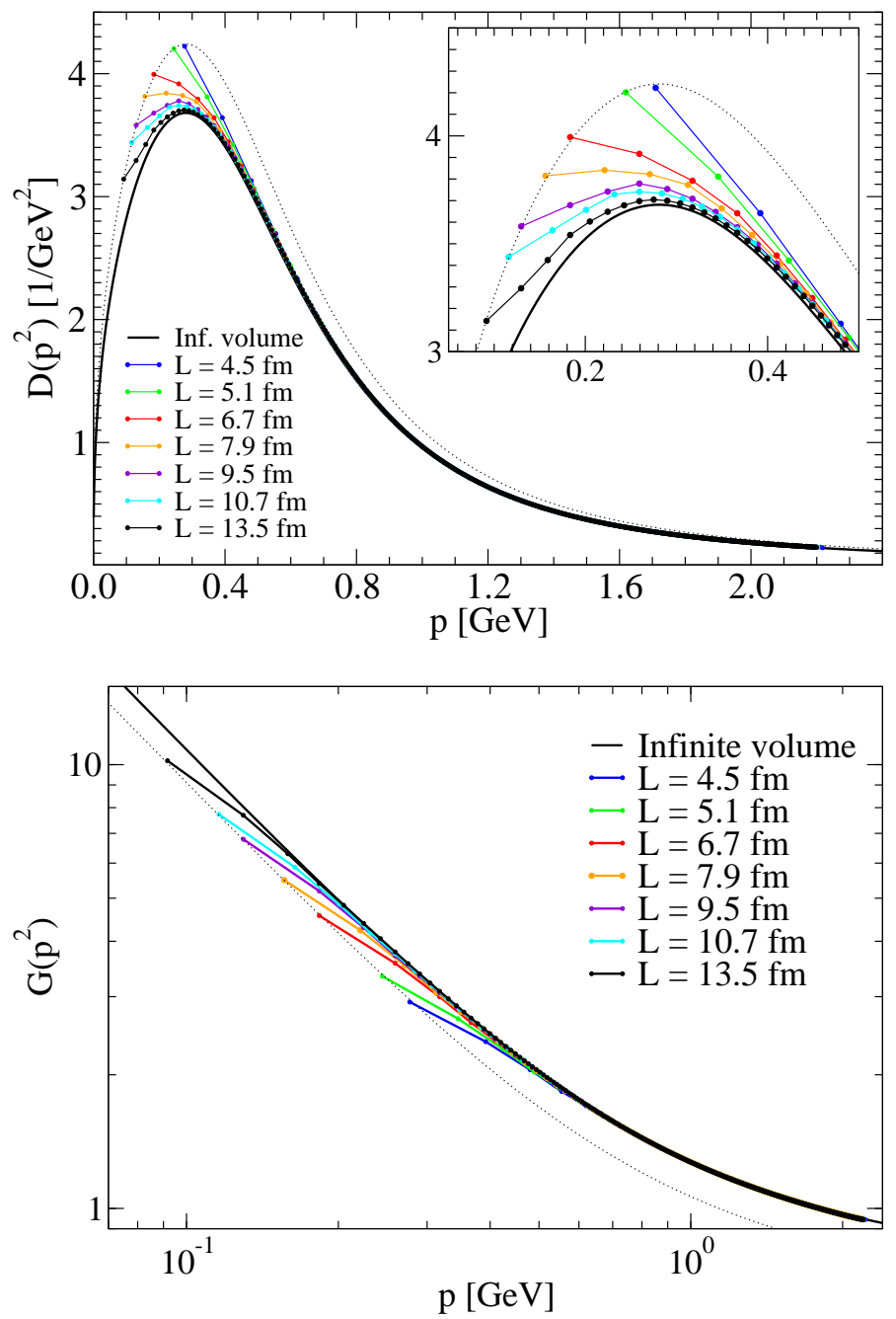

Figure 1: Numerical solutions on tori with different volumes compared to the infinite volume limit. The upper graph shows the gluon propagator, whereas on the lower graph the ghost dressing function is plotted.

The corresponding numerical results on different volumes are shown in Figure 1. The momentum scale is fixed by comparison with corresponding lattice calculations, see [22] for details. We discuss results on seven different volumes $V=L^{4}$; the corresponding box lengths $L$ are given in the legends of Figure 1. One clearly observes that the infinite volume solutions of the gluon propagator $D\left(p^{2}\right)$ and ghost dressing function $G\left(p^{2}\right)$ are more and more approached by the torus solutions with increasing volume. Qualitatively one can see the following behaviour: the gluon 

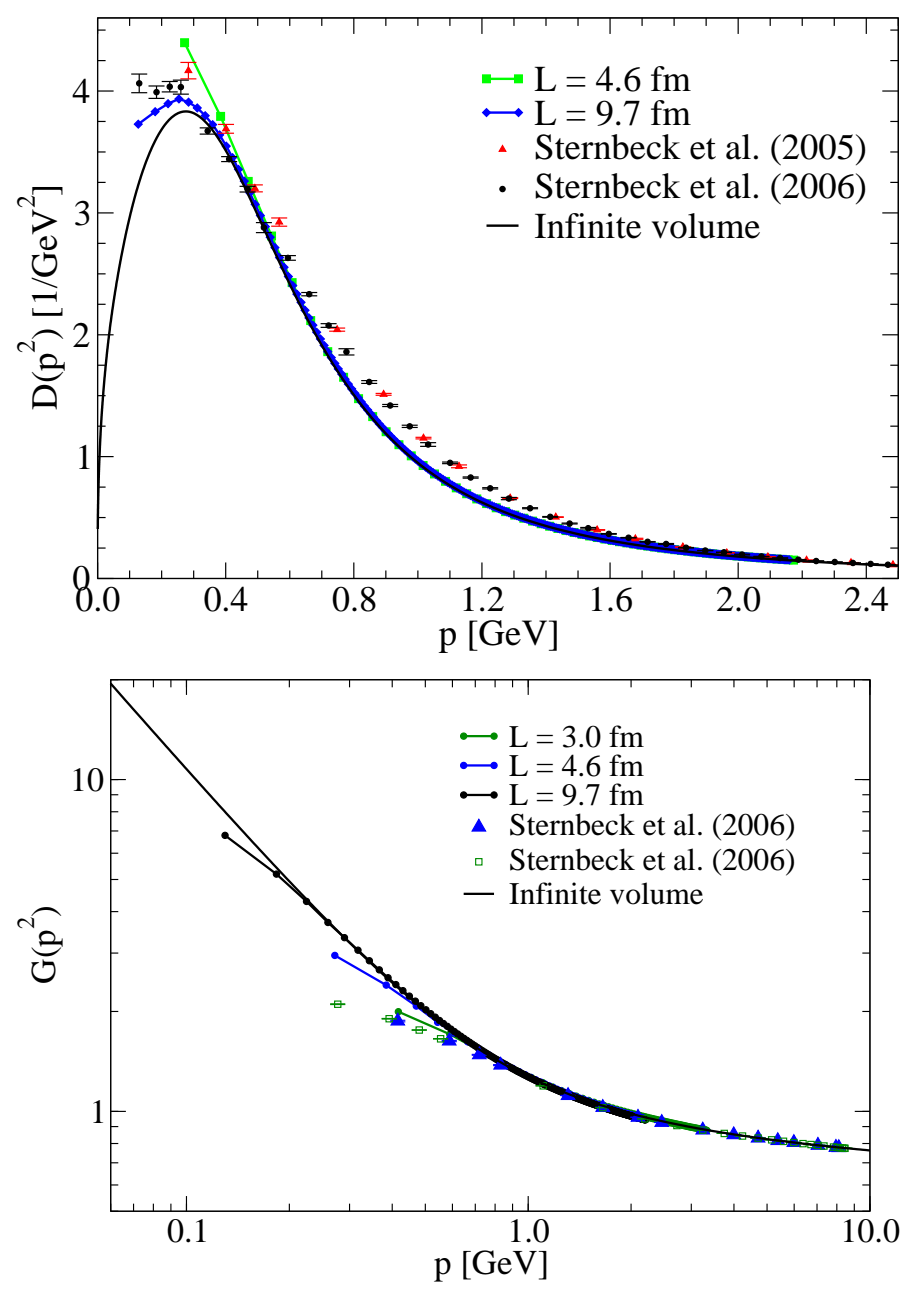

Figure 2: DSE results for the gluon propagator and ghost dressing function on tori with different volumes compared to recent lattice calculations on similar manifolds. The lattice data are taken from Refs. [20, 17].

propagator seems to be divergent at volumes of $V \approx(4-8 \mathrm{fm})^{4}$. For larger volumes the propagator bends downwards to reach a plateau at roughly $V \approx(9 \mathrm{fm})^{4}$ and at $V \approx(10 \mathrm{fm})^{4}$ the propagator is infrared vanishing and therefore qualitatively similar to the infinite volume limit given in (1.2). For the ghost we observe that the first two points on each volume bend away from the power law behaviour of the infinite volume solution. With increasing volume more and more of the remaining points are in the 'scaling region', where the infinite volume power law develops.

We now compare our results from DSEs on a torus to the ones from lattice calculations. Nowadays, lattice data for the gluon propagator are available on impressively large lattices. The authors of [12] report on an $S U(2)$-study on a $52^{4}$-lattice, whereas in [20] results from an $S U$ (3) calculation on a $56^{4}$-lattice are discussed. Results on even larger lattices have been presented at this conference $[27,28]$. In the upper graph of Figure 2 we display the $S U$ (3)-results of [20] together with data on a smaller volume [17] and compare with DSE-results on tori with similar volumes.

The qualitative agreement of the solutions at similar volumes in the infrared is interesting. ${ }^{1}$

\footnotetext{
${ }^{1}$ Differences in the intermediate momentum regime at approximately $1 \mathrm{GeV}$ are truncation artefacts of the DSE
} 
Whereas both, the lattice and the DSE result at the smaller volume $V \approx(4.6 \mathrm{fm})^{4}$ seem to diverge, one starts to observe an infrared finite one, or perhaps even a slight infrared suppression, at the larger volumes $V \approx(9.7 \mathrm{fm})^{4}$. This indicates that the scaling behaviour of the lattice results with volume may be similar to the ones of the DSE solution. If this is correct one should see a turnover of the gluon propagator at even larger volumes.

The situation is far less clear for the ghost dressing function. Our results for three different volumes, $V=(3.9,4.6,9.7 \mathrm{fm})^{4}$, are compared to the $\mathrm{SU}(3)$ lattice results of [20, 17]. For the DSE solutions we observe a characteristic deviation of the two lowest momentum points at each volume from the infinite volume solution, corresponding to a ghost mass which goes to zero in the infinite volume limit [22]. The lattice results do not seem to show such behaviour as yet. Even though the lattice volumes herein are roughly between $3 \mathrm{fm}$ and $4.5 \mathrm{fm}$, and thus still rather small, there appears to be not much sign of a volume dependence at all at this point. However, it has been observed that effects at intermediate momenta can in a subtle way influence the finite-volume effects in the ghost propagator [29]. As these are truncation-dependent in the DSEs, this may explain the difference to the results in lattice calculations.

\section{Epilogue}

In this conference first results have been presented for the gluon propagator on very large lattices $\left(128^{4}\right.$ [27] and $112^{4}$ [28]). Both groups do not see a turnover of the gluon propagator, even though the volumes exceed $(10 \mathrm{fm})^{4}$ by far. Does this mean that the gluon propagator is finite in the infrared, i.e. $\kappa=0.5$ ? In principle there is not much to say against this scenario from the pure DSE/FRGE-perspective. It could be that the IR-regular dressing of the nonperturbative ghost-gluon vertex provides the necessary corrections to drive the IR-anomalous dimension towards $\kappa \cong 0.5$. However, it is an unambiguous and truncation independent prediction of the Dyson-Schwinger and the functional renormalisation group framework that the ghost dressing function should then diverge as $G\left(p^{2}\right) \sim\left(p^{2}\right)^{-0.5}$ [8]. Current lattice results in four dimensions do not seem to support this relation. On the other hand, lattice data in two dimensions [29] agree well with the corresponding DSE-results [30]. The usual suspects for the remaining discrepancy in four dimensions then being gauge fixing or renormalisation problems. Gauge fixing algorithms are known to be less efficient at larger lattices where the number of Gribov copies increases exponentially. Naturally this effect also depends on the number of dimensions. Furthermore, it is also known, that effects from Gribov copies influence the ghost propagator much stronger than the glue [17, 19]. Finally, very recent lattice results allowing a wider class of gauge transformations in the minimisation procedure [13] tend to result in an additional suppression of the gluon propagator at low momenta, which is observed already in rather moderate volumes of $V=(6.5 \mathrm{fm})^{4}$.

Also in the continuum one probably needs additional verification for Zwanziger's idea that gauge fixing on the first Gribov region (as done in DSEs and FRGEs) is enough to avoid effects from Gribov copies [4]. This idea underlies all continuum studies of infrared anomalous dimensions so far. Therefore, the final settlement of the infrared behaviour of ghost and glue awaits further clarification in both, the continuum and lattice studies.

solutions. One can show analytically that this is the only region where the omitted gluonic two-loop diagrams contribute significantly. 


\section{Acknowledgements}

CF would like to thank the organisers of Lattice 2007 for all their efforts which made this inspiring conference possible. We are grateful to A. Cucchieri, T. Mendez, M. Müller-Preussker, O. Oliveira, A. Sternbeck and A. Williams for interesting discussions. This work was supported by the Helmholtz-University Young Investigator Grant VH-NG-332, by the DFG under grant no. MA-3935/1-2 and Al 279/5-2, and by the Australian Research Council.

\section{References}

[1] T. Kugo and I. Ojima, Prog. Theor. Phys. Suppl. 66 (1979) 1 [Erratum Prog. Theor. Phys. 71, 1121 (1984)]. T. Kugo, hep-th/9511033.

[2] V. N. Gribov, Nucl. Phys. B 139, 1 (1978); D. Zwanziger, Nucl. Phys. B 412, 657 (1994).

[3] R. Alkofer and L. von Smekal, Phys. Rept. 353 (2001) 281; C. S. Fischer, J. Phys. G 32 (2006) R253.

[4] D. Zwanziger, Phys. Rev. D 65 (2002) 094039; Phys. Rev. D 69, 016002 (2004).

[5] J. Gattnar, K. Langfeld and H. Reinhardt, Phys. Rev. Lett. 93 (2004) 061601;

[6] L. von Smekal, R. Alkofer and A. Hauck, Phys. Rev. Lett. 79 (1997) 3591.

[7] R. Alkofer, C. S. Fischer and F. J. Llanes-Estrada, Phys. Lett. B 611, 279 (2005).

[8] C. S. Fischer and J. M. Pawlowski, Phys. Rev. D 75 (2007) 025012.

[9] Ch. Lerche and L. von Smekal, Phys. Rev. D 65 (2002) 125006.

[10] J. M. Pawlowski, D. F. Litim, S. Nedelko and L. von Smekal, Phys. Rev. Lett. 93 (2004) 152002; AIP Conf. Proc. 756 (2005) 278.

[11] A. Cucchieri, A. Maas and T. Mendes, Phys. Rev. D 75 (2007) 076003 [arXiv:hep-lat/0702022].

[12] A. Cucchieri and T. Mendes, Braz. J. Phys. 37 (2007) 484 [arXiv:hep-ph/0605224]; and references therein.

[13] I. L. Bogolubsky, V. G. Bornyakov, G. Burgio, E. M. Ilgenfritz, M. Muller-Preussker and V. K. Mitrjushkin, arXiv:0707.3611 [hep-lat].

[14] P. O. Bowman, U. M. Heller, D. B. Leinweber, M. B. Parappilly, A. Sternbeck, L. von Smekal, A. G. Williams and J. Zhang, arXiv:hep-lat/0703022.

[15] P. O. Bowman, U. M. Heller, D. B. Leinweber, M. B. Parappilly and A. G. Williams, Phys. Rev. D 70 (2004) 034509 [arXiv:hep-lat/0402032].

[16] O. Oliveira and P. J. Silva, arXiv:0705.0964 [hep-lat].

[17] A. Sternbeck, E. M. Ilgenfritz, M. Mueller-Preussker and A. Schiller, Phys. Rev. D 72 (2005) 014507.

[18] E. M. Ilgenfritz, M. Muller-Preussker, A. Sternbeck and I. L. Bogolubsky, Braz. J. Phys. 37 (2007) 193 [arXiv:hep-lat/0609043]; A. Sternbeck, PhD Thesis, Humboldt University Berlin, hep-lat/0609016.

[19] O. Oliveira and P. J. Silva, Eur. Phys. J. A 31 (2007) 790 [arXiv:hep-lat/0609027].

[20] A. Sternbeck, E. M. Ilgenfritz, M. Müller-Preussker, A. Schiller and I. L. Bogolubsky, PoS LAT2006 (2006) 076.

[21] C. S. Fischer, R. Alkofer and H. Reinhardt, Phys. Rev. D65 (2002) 094008; C. S. Fischer, B. Grüter and R. Alkofer, Annals Phys. 321 (2006) 1918.

[22] C. S. Fischer, A. Maas, J. M. Pawlowski and L. von Smekal, Annals of Physics arXiv:hep-ph/0701050.

[23] P. Watson and R. Alkofer, Phys. Rev. Lett. 86 (2001) 5239.

[24] W. Schleifenbaum, A. Maas, J. Wambach and R. Alkofer, Phys. Rev. D 72 (2005) 014017.

[25] A. Maas, A. Cucchieri and T. Mendes, Braz. J. Phys. 37N1B (2007) 219 [arXiv:hep-lat/0610006];

A. Cucchieri, T. Mendes and A. Mihara, JHEP 0412 (2004) 012.

[26] C. S. Fischer and R. Alkofer, Phys. Lett. B536 (2002) 177.

[27] A. Cucchieri and T. Mendes, these proceedings.

[28] A. Sternbeck, D. B. Leinweber, L. von Smekal and A. G. Williams, these proceedings.

[29] A. Maas, Phys. Rev. D 75 (2007) 116004.

[30] M. Huber, R. Alkofer, C. S. Fischer and K. Schwenzer, arXiv:0705.3809 [hep-ph]. 